\title{
Health promotion concepts in aging
}

Health Promotion (HP) has been gaining prominence in the context of WHO, which can be better understood in the light of a historical perspective (Alma Ata declaration 1978, Health for All Movement, 1982, Ottawa Charter 1986).

This constant evolution in the concept and extend of HP has triggered a general awareness for new perspectives within and beyond the health sector, incorporating a wide range of segments of society and disciplines towards a same objective.

As a vulnerable high risk group, the most increasing segment of the population and the major consumers of health services, old people have become an important target group for HP strategies, whit the possibility of further compressing the period at the end of life cyrcle during which the greatest mortality, morbidity and health care costs occur.

However, there is a great deal about what may be reasonable aplying to old people. There are still deficiences in the knowledge base on HP in the older population. A major problem has been the lack of epidemiologic data in older population and the abstance of evaluated interventions. The exclusion of elderly individuals from studies is often based on the belief that the treatment or prevention products few benefits beyond a certain age.

The focus of HP in the younger persons is clearly preventive. The changing nature of illness risks and health maintenance needes over the life course suggest a broader focus for older adults. The overall goal of HP in the elderly should help older persons mantain their functional independence.

An undesrtanding of the multiple factors affecting the health of the elderly, the distinction between HP and ageing and HP in old age are necessary concepts for the planning of HP interventions to this age group.

The delivery of information and the training of health professionals are important areas of action.

Healthy ageing is becoming an important area for research. It reflects the shift from the "disablement-handicap", common association with older people, to a potentialy enabling positive view of old age, more coherent with the HP concept. Sucessful examples of interventions are needed. 